\title{
Estudio de los mecanismos de degradación de la interfase acero (limpio u oxidado)- recubrimiento clorocaucho en su exposición al ensayo de condensación de humedad
}

\author{
B. Chico ${ }^{(*)}$ y S. Feliu $(\mathrm{Jr} .)^{(*)}$
}

\begin{abstract}
Resumen En el presente trabajo se analiza el efecto de la exposición a la humedad de un recubrimiento orgánico de clorocaucho aplicado a un sustrato de acero (únicamente limpio o previamente oxidado) sobre los valores de adherencia del sistema y la composición química de la interfase metal-pintura. Se ha intentado establecer posibles relaciones entre la degradación de la interfase acero-recubrimiento clorocaucho en su exposición a condiciones de alta humedad y la presencia o ausencia de una capa de óxido sobre el sustrato original. Después de 1 día de exposición a la humedad, se observó un aumento en los valores de adherencia del sistema pintura-acero sin oxidar. Sorprendentemente, estos valores alcanzan un máximo después de 15 días de exposición. Este mismo comportamiento se había encontardo ya en el sistema acero preoxidado-recubrimiento clorocaucho. Tiempos de exposición a la humedad suficientemente largos (30-60 días) producen una acusada disminución en los valores de adherencia y un desplazamiento del lugar donde ocurre el fallo desde el seno de la pintura a la región interfacial entre la pintura y el sustrato.
\end{abstract}

Palabras clave: Recubrimiento clorocaucho. Degradación interfacial. Adherencia. Espectroscopía fotoelectrónica de rayos X (XPS).

\section{Study of the mechanisms of humidity-induced adhesion loss of chlorinated rubber on cleaned and rusted steel substrates}

\begin{abstract}
The effect of time of exposure to the humidity on the behaviour of the system chlorinated rubber paint-steel substrate (cleaned or pre-rusted) is investigated from the interfacial chemistry and adhesion points of view. After one day of humidity exposure, the paint-rust free steel substrate system shows an increase of adhesion. A maximum adhesion value is obtained after 15 days of exposure. This behaviour is similar to that previously described for a pre-rusted steel substrate. After 30-60 days of exposure, the adhesion strength values show an important reduction and the locus of adhesion loss shifts from the paint inside to the interphase between the metallic substrate and the paint.
\end{abstract}

Keywords: Chlorinated rubber. Interfacial degradation. Adhesion. X-ray photoelectron spectroscopy (XPS).

\section{INTRODUCCIÓN}

En un estudio previo (1), se observó que al aplicar un recubrimiento clorado a una superficie oxidada de acero se producía rápidamente la degradación del sistema metal-pintura en la exposición

(*) Centro Nacional de Investigaciones Metalúrgicas, CENIM (CSIC), Avda. de Gregorio del Amo, 8. 28040-Madrid (España). Fax (91) 5347425 atmosférica, que se apreciaba visualmente por la aparición de manchas de óxido y fallos de adherencia. Esta degradación era independiente del índice de agresividad de las diferentes atmósferas en las que se habían expuesto las probetas. Tampoco se detectaron contaminantes salinos retenidos en la capa de óxido inicial que pudiera llevar a la rápida degradación del sistema. En este trabajo se ha tratado de obtener información adicional sobre el efecto del tiempo de exposición y del grado de oxidación del sustrato de acero sobre el que se aplica 
el recubrimiento de pintura clorocaucho. Se han utilizado como sustratos: $a$ ) acero bajo en carbono simplemente limpio por ultrasonidos y $b$ ) acero preoxidado (expuesto 1 mes en condiciones de alta humedad relativa). Con la ayuda de la espectroscopía fotoelectrónica de rayos X (XPS) y recurriendo, además, a ensayos de adherencia, se ha intentado establecer posibles relaciones entre la degradación de la interfase acero-recubrimiento de pintura clorocaucho en su exposición a condiciones de alta humedad y el estado de oxidación del sustrato de acero de partida.

\section{PARTE EXPERIMENTAL}

La pintura, los sustratos metálicos, el ensayo de condensación de humedad, los dispositivos experimentales y las condiciones utlizadas para la obtención de los espectros XPS están descritos en profundidad en un trabajo anterior (2).

\section{RESULTADOS}

\subsection{Análisis por XPS de las superficies de pintura interfaciales}

La tabla I, extraida de los espectros generales XPS, muestra el porcentaje elemental determinado en las superficies correspondientes al seno del recu-

TABla I.- Composición atómica de la superficie de pintura del sistema metal-pintura sin exponer y después de su exposición en condiciones de alta humedad

TABLE I.- Atomic compositions of the interfacial paint surfaces for the non-exposed metal/paint system and after exposure to the condensing humidity test

\begin{tabular}{|llllll|}
\hline Muestra & $\% \mathrm{C}$ & $\% \mathrm{O}$ & $\% \mathrm{Cl}$ & $\% \mathrm{Si}$ & $\% \mathrm{~Pb}$ \\
\hline $\begin{array}{l}\text { Superficie de pintura Clorocaucho } \\
\text { (Superficies de Referencia) }\end{array}$ & & & \\
\hline $\begin{array}{l}\text { Seno del } \\
\text { recubrimiento }\end{array}$ & 54 & 22 & 16 & 5 & 3 \\
\hline $\begin{array}{l}\text { Superficie de } \\
\text { pintura interfacial } \\
\text { (sin exponer) }\end{array}$ & 69 & 12 & 17 & 1.5 & 0.5 \\
\hline Superficies de pintura interfaciales después de exponer \\
\hline 1 día & 70 & 12 & 15 & 2.5 & 0.5 \\
\hline 15 días & 64 & 18 & 14 & 3.5 & 0.5 \\
\hline 30 días & 71 & 11 & 16 & 2 & Trazas \\
\hline 60 días & 70 & 9 & 19 & 1.5 & Trazas \\
\hline
\end{tabular}

brimiento de pintura, en la superficie de pintura interfacial sin exponer (ambas superficies de pintura utilizadas como referencias) y después de exponer al ensayo de condensación de humedad. La superficie de pintura correspondiente al seno del recubrimiento presenta un mayor contenido en pigmento (silicio y plomo). También se observa un mayor contenido de oxígeno y un menor contenido de carbono respecto a las otras superficies de pintura interfacial. Después de 15 días de ensayo, se observa, en la superficie de pintura interfacial, contenidos de carbono, oxígeno y cloro similares a los obtenidos en la superficie de pintura correspondiente al seno del recubrimiento. Después de tiempos de ensayo suficientemente largos (30-60 días), la composición de las superficies de pintura interfaciales tienden a parecerse a las superficies interfaciales de pintura sin ensayar o al inicio del ensayo.

\subsection{Análisis por XPS de las superficies del sustrato interfacial}

El porcentaje elemental determinado en las superficies correspondientes al acero sin exponer, la superficie del sustrato interfacial sin exponer (ambas superficies metálicas utilizadas como referencia) y después de exponer al ensayo de condensación de humedad, se reflejan en la tabla II. Después de 1 día de exposición, la señal del hierro en el espectro XPS prácticamente ha desaparecido. Visualmente, el sustrato metálico aparecía prácticamente cubierto de pintura, aunque se observaba con claridad la presencia de restos de metal del sustrato original. El análisis por XPS de la superficie correspondiente al sustrato interfacial obtenido después

TABLA II.- Composición atómica de la superficie del sustrato del sistema metal-pintura sin exponer y después de su exposición en condiciones de alta humedad

TABLE II.-Atomic compositions of the interfacial steel substrates surfaces for the non-exposed metal/paint system and after exposure to the condensing humidity test

\begin{tabular}{|c|c|c|c|c|c|c|}
\hline Muestra & $\% \mathrm{C}$ & $\% \mathrm{O}$ & $\% \mathrm{Fe}$ & $\% \mathrm{Cl}$ & $\% \mathrm{Si}$ & $\% \mathrm{~Pb}$ \\
\hline Acero original & 70 & 26 & 4 & 0 & 0 & 0 \\
\hline \multicolumn{7}{|c|}{$\begin{array}{l}\text { Superficies del sustrato metálico interfacial después } \\
\text { de exponer }\end{array}$} \\
\hline Sin exponer & 67 & 15 & 1 & 15 & 2 & Trazas \\
\hline 1 día & 69 & 13 & Trazas & 17 & 3 & Trazas \\
\hline 15 días & 60 & 21 & 0 & 14 & 4 & Trazas \\
\hline 30 días & 68 & 13 & 1 & 16 & Trazas & Trazas \\
\hline 60 días & 69 & 12 & 1 & 17 & Trazas & Trazas \\
\hline
\end{tabular}


de 15 días de exposición a la humedad muestra una composición similar a las observadas en la superficie correspondiente al seno del recubrimiento y la de pintura interfacial después de 15 días de exposición (Tabla I). Visualmente, la superficie del substrato interfacial aparecía completamente cubierto de pintura clorocaucho. Después de prolongados períodos de exposición a la humedad (30-60 días), vuelve a aparecer un cierto contenido de hierro y la señal del silicio prácticamente ha desaparecido, quizás en coincidencia con el deterioro de la interfase metal-pintura. Visualmente, aparecían productos de corrosión en el sustrato metálico interfacial.

\subsection{Medidas de adherencia}

En la figura 1 se muestra la evolución de los valores de adherencia con el tiempo para el sistema recubrimiento de clorocaucho-acero ensayado en la cámara de condensación de humedad. Después de 1 día de exposición, se observó un pequeño aumento en los valores de adherencia; sorprendentemente, estos valores alcanzan un máximo después de 15 días de exposición. Al cabo de unos treinta días, se producía una brusca disminución, directamente relacionada con el deterioro de la interfase.

También, en la figura 1, se muestra la variación del porcentaje de silicio determinado en la superficie del sustrato interfacial con el tiempo. El porcentaje de silicio pasa por un máximo después de 15 días de exposición, tendiendo a decaer a continuación. Tiene interés hacer notar en la figura 1, la

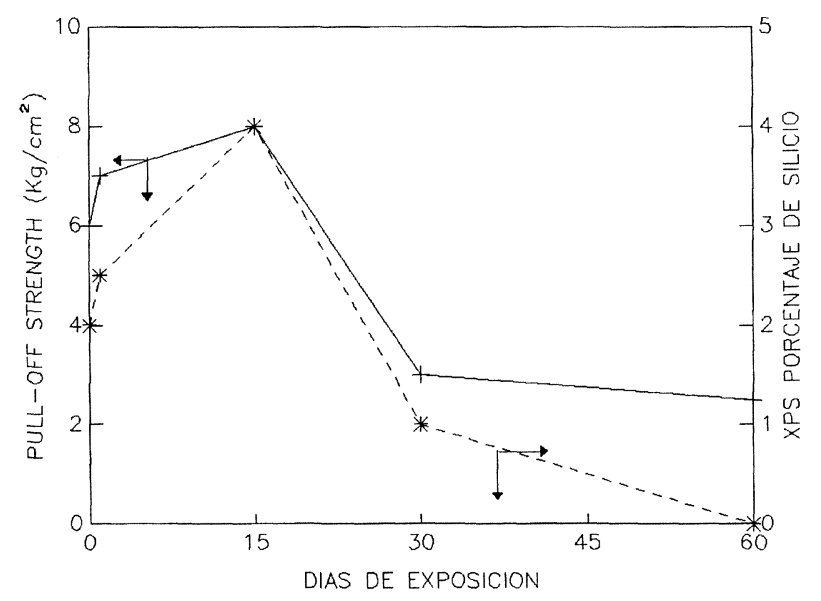

FIG. 1.- Evolución de los valores de adherencia (+) y el porcentaje atómico de silicio (*) observado en la superficie del sustrato con el tiempo de exposición al ensayo de condensación de humedad.

FIG. 1.- Evolution of the adhesion strength values $+(+)$ and XPS silicon atomic percentages on the interfacial substrate surface (*) with the time of humidity exposure. estrecha relación entre los valores de adherencia y el porcentaje de silicio determinado en el sustrato interfacial, confirmación de que ambos parámetros dependen de un mismo fenómeno: esto es, fortalecimiento de la interfase metal-pintura a tiempos cortos de exposición (15 días) y desplazamiento del lugar donde ocurre el fallo bajo tensión desde la interfase metal-pintura al seno del recubrimiento.

\section{DISCUSIÓN}

A partir de los resultados obtenidos en este estudio, se observa que, en los sustratos de acero no oxidado, los valores de adherencia del sistema acero-recubrimiento clorocaucho dependen del tiempo de exposición en condiciones de alta humedad. A esta misma conclusión se había llegado con los sustratos de acero previamente oxidados (2).

Los valores de adherencia en el sistema acero no oxidado son inicialmente elevados (Fig. 1). Visualmente, aparecía el sustrato metálico interfacial resultante del pull-off test, prácticamente cubierto de pintura con algún resto del sustrato metálico. En los datos XPS de la interfase metal-pintura, se observa una importante concentración de silicio y cloro (elementos propios de la pintura) en la superficie metálica interfacial (Tabla II). Estos datos permiten afirmar que al someter el sistema a tracción, el fallo ocurre no en la interfase metal-pintura, sino en las capas de pintura más cercanas al sustrato metálico (fallo cohesivo). Con el sustrato de acero previamente oxidado (2), los datos XPS del sistema sin exponer indicaban que el fallo bajo tensión ocurría en la capa de herrumbre más cercana a la pintura y se obtenían valores de adherencia más bajos.

Debe ponerse de relieve el incremento de los valores de adherencia a tiempos cortos de ensayo en el sistema acero no oxidado-pintura clorocaucho contrariamente a lo que generalmente ocurre en los sistemas metal-pintura (3). Este mismo comportamiento se había encontrado ya en el sistema acero preoxidado-recubrimiento clorocaucho (2).

Debe ponerse de relieve el incremento del contenido de silicio en ambas superficies interfaciales y la ausencia de hierro en la superficie del sustrato interfacial después de 15 días de exposición a la humedad (Tablas I y II). Con los sustratos de acero preoxidados, se había encontrado que la cantidad de pintura sobre el sustrato interfacial tendía a aumentar a cortos tiempos de exposición, lo que sugería un movimiento del lugar donde ocurría el fallo bajo tensión desde la interfase herrumbre-pintura hacía una región de pintura cercana al sustrato (2). Este comportamiento parece repetirse en el sistema acero no oxidado-recubrimiento clorocaucho a cortos períodos de exposición a la humedad. 
Este ensayo permite afirmar que el sistema recubrimiento clorocaucho-acero tiene una gran tendencia a fortalecer la resistencia mecánica de su interfase a cortos períodos de exposición a la humedad, independientemente del estado de oxidación del sustrato de acero sobre el que se aplique la pintura. Feliu et al. (4) llegaron a la conclusión de que, independientemente del sustrato metálico sobre el que se aplique el recubrimiento clorocaucho y como resultado del proceso de curado, se produce una capa de débil resistencia mecánica (weak boundary layer) constituida fundamentalmente por carboxilatos del metal. En el presente estudio, se podría especular que, como resultado de la exposición del sistema en condiciones de alta humedad, se produce la disolución de esta capa salina de carboxilatos (de débil resistencia mecánica) motivando el aumento de los valores de adherencia del sistema.

Tiempos de exposición a la humedad suficientemente largos (30-60 días) producen una acusada disminución de los valores de adherencia determinados en el sistema acero no oxidado-recubrimiento clorocaucho. Esta misma disminución se había observado con el sustrato de acero previamente oxidado. Visualmente, aparecen signos de degradación en el recubrimiento de pintura (su color cambia de rojo a rojo-amarillo).

Como se muestra en la tabla II, después de 30 y 60 días de exposición a la humedad, se observa un incremento de la cantidad de hierro detectada en el sustrato metálico interfacial comparada con las cantidades obtenidas después de 1 y 15 días de exposición. Debe destacarse que las superficies de pintura interfaciales no presentan restos de metal $\mathrm{u}$ óxidos a lo largo del tiempo de ensayo (Tabla 1). Estos datos sugieren que el fallo, al someter el sistema acerorecubrimiento clorocaucho después de una prolongada exposición a la humedad, tiene lugar en la región de pintura más cercana al sustrato. En un trabajo anterior (2), con sustratos de acero preoxidados, se observó una cierta cantidad de hierro en la superficie interfacial de pintura después de 30 días de exposición. Esta cantidad aumentó después de 60 días de exposición.

La ausencia de una importante degradación química en las superficies de pintura interfaciales después de un período de exposición de 30-60 días sugieren que el probable mecanismo de pérdida de adherencia sea de naturaleza mecánica. Durante la exposición al medio agresivo, el crecimiento de la capa de productos de corrosión del hierro provocaría la creación de tensiones laterales muy elevadas en la intercara metal-pintura que darían lugar a la disminución en los valores de adherencia. En el sistema acero herrumbrado-recubrimiento clorocaucho el mecanismo de pérdida de adherencia sería parecido teniendo en cuenta que la actividad catódica del sustrato de acero previamente oxidado es mayor que la del acero no herrumbrado.

\section{CONCLUSIONES}

Mediante una combinación de medidas por XPS y de adherencia, se ha encontrado que la adherencia del sistema acero sin oxidar-recubrimiento de pintura clorocaucho depende del período de exposición. La variación de los valores de adherencia con el período de exposición es prácticamente independiente de la presencia o ausencia de una capa de herrumbre en el sustrato de acero inicial. Al comienzo del ensayo (hasta 15 días), se observa un incremento en los valores de adherencia. Como la misma variación se observó en el sustrato de acero preoxidado, parece que en este incremento apenas influye el grado de oxidación del sustrato. Los resultados obtenidos sugieren que este incremento de adherencia está determinado por la disolución de la capa de carboxilatos de hierro $(W B L)$ que se forma, resultado del proceso de curado de los recubrimientos clorocaucho.

Destaca la disminución de los valores de adherencia después de largos períodos de exposición (30-60 días). Probablemente, el engrosamiento de la capa de productos de corrosión del hierro sobre el sustrato metálico se traduce en una disminución de la resistencia mecánica de la región interfacial.

\section{REFERENCIAS}

(1) Morcillo, M., Simancas, J., Fierro, J.L.G., Feliu, S. (Jr.) y Galvan, J.C. Prog. Org. Coat., 21, 1993: 315.

(2) Feliu, S. (Jr.), Fierro, J.L.G. y Maffiotte, C. Prog. Org. Coat. 30, 1997: 247.

(3) Walker, P. Paint. Technol., 31 (8), 1967: 22.

(4) Feliu, S. (Jr.) y Chico, B. Comunicación enviada a Surf. Interf. Anal. 